\title{
Filamentous fungal endophthalmitis: results of combination therapy with intravitreal amphotericin B and voriconazole
}

This article was published in the following Dove Press journal:

Clinical Ophthalmology

13 April 2015

Number of times this article has been viewed

\author{
Kopal Mithal' \\ Avinash Pathengay' \\ Abhishek Bawdekar' \\ Animesh Jindal' \\ Divya Vira ${ }^{2}$ \\ Nidhi Relhan ${ }^{3}$ \\ Himadri Choudhury' \\ Namrata Gupta' \\ Varun Gupta' \\ Nagendra K Koday ${ }^{4}$ \\ Harry W Flynn Jr ${ }^{3}$
}

'Retina and Uveitis Services, ${ }^{2}$ Cornea Services, LV Prasad Eye Institute, GMR Varalakshmi Campus, Visakhapatnam, India; ${ }^{3}$ Department of Ophthalmology, Bascom Palmer Eye Institute, University of Miami Miller School of Medicine, Miami, FL, USA; ${ }^{4}$ Ocular Microbiology Service, LV Prasad Eye Institute, GMR Varalakshmi Campus, Visakhapatnam, India
Correspondence: Avinash Pathengay

L V Prasad Eye Institute GMR

Varalakshmi Campus, II-II3/I,

Hanumanthawaka Junction,

Visakhapatnam 530040 Andhra Pradesh, India

Tel +9| 89| 39892020

Fax +918913984444

Email avinash@lvpei.org
Purpose: To report outcomes of exogenous fungal endophthalmitis treated with combination of intravitreal antifungal agents.

Design: Retrospective, non-randomized, interventional, consecutive case series.

Methods: Twelve eyes of twelve consecutive cases of filamentous fungal endophthalmitis were treated with a combination of intravitreal amphotericin-B and intravitreal voriconazole (AmB-Vo Regime) along with pars plana vitrectomy at a single center. Clinical characteristics, microbiology results, treatment strategy, visual, and anatomical outcomes were analyzed.

Results: Ten cases out of the twelve were postoperative endophthalmitis of which nine were part of a post cataract surgery cluster. The remaining included endophthalmitis following keratitis post pterygium excision (1) and following open globe injury (2). The most common fungus was Aspergillus terreus, which was isolated in 8/12, followed by A. flavus in 2/12 and Fusarium solani in $1 / 12$. The presenting visual acuity ranged from light perception (LP) to counting fingers. The visual acuity at final follow-up was $20 / 400$ or better in $7 / 12$ eyes $(58.33 \%)$ and 20/60 in 2/12 eyes (range 20/60 to LP). All eyes with corneal involvement had final visual acuity 20/400 or worse. Globe salvage was achieved in all cases.

Conclusion: Combining intravitreal amphotericin-B and voriconazole could be a novel treatment strategy in the management of endophthalmitis caused by filamentous fungus. Eyes with corneal involvement had poor visual outcome either with or without therapeutic penetrating keratoplasty.

Keywords: fungal, endophthalmitis, Aspergillus, intravitreal, voriconazole, amphotericin B

\section{Introduction}

Endophthalmitis caused by filamentous fungi has high ocular morbidity and is associated with a poor visual outcome. Recovery of 20/400 or better vision is reported in less than $50 \%$ of patients, while up to $60 \%$ undergo enucleation. ${ }^{1,2}$ Limitations of antifungals used in the management of filamentous fungal endophthalmitis include the fungistatic nature of the drugs, poor intraocular penetration of topical and systemic antifungals, development of resistance to available antifungal drugs, and lack of routine susceptibility testing of fungal isolates. ${ }^{3,4}$ Thus, current antifungals could fail to eradicate the disease when used as monotherapy. Combining drugs like amphotericin B and voriconazole, which have complementary mechanisms of action, has been reported in the successful management of refractory systemic Aspergillus infections. ${ }^{5}$ However, there are reports of disparity in results for the in vivo and in vitro effects of combining the two different groups of antifungal drugs. ${ }^{6,7}$ We report our experience with use of a combination of intravitreal amphotericin B and voriconazole in the management of 12 consecutive cases of filamentous fungal endophthalmitis. 


\section{Patients and methods}

This was a retrospective study of 12 consecutive endophthalmitis cases caused by filamentous fungi that were managed at our center between January 2013 and August 2013. All patients treated with a combination of intravitreal amphotericin B and voriconazole were included. Medical records were reviewed in accordance with the guidelines laid down by the Declaration of Helsinki. Institutional review board approval was obtained. The data collected consisted of demographic details, affected eye, etiology, duration of symptoms, clinical characteristics, visual acuity and intraocular pressure at presentation and at subsequent follow-up. The collected samples, which included corneal scrapings, vitreous biopsy, and explanted intraocular lens, were plated in both bacterial and fungal culture media. Microbiology records were reviewed for intraocular samples tested, direct microscopy results, and culture characteristics. The details of the treatment course, including time and number of surgical interventions, along with type, number, route, and duration of use of antifungals, were recorded (Table 1).

\section{Results}

Twelve consecutive cases of filamentous fungal endophthalmitis were included in this single-center case series. The mean age of the patients was 50.75 (range 19-64) years. Details of demographic features, clinical characteristics, microbiology results, treatment, and clinical outcome for all cases are described in Table 1. The mean time since onset of symptoms was 3.2 (range 1-7) days. The patients presented to us at a median 22 (mean 28.4, range 22-62) days postoperatively. Initial visual acuity in all eyes was finger counting at 2 feet or worse. Nine post cataract surgery endophthalmitis cases reported onset of symptoms within one week of surgery. However, patients originating from a remote rural area presented with a delay of 21-62 days after surgery. Two endophthalmitis cases occurred following open globe injury and one following keratitis after pterygium excision. The presenting complaint was a sudden-onset decrease in visual acuity in eleven eyes (91.6\%) associated with pain in eight eyes (66.6\%). Four eyes had associated corneal or scleral tunnel infiltrate, and fungal hyphae were demonstrated on microscopy for all of these. Six eyes (50\%) presented with hypopyon or anterior chamber exudates, while all eyes had exudates in the vitreous cavity that were either seen clinically or demonstrated on B-scan ultrasonography. All eyes underwent pars plana vitrectomy with injection of antifungals, comprising daily intravitreal voriconazole $(100 \mu \mathrm{g} / 0.1 \mathrm{~mL})$ and alternate day intravitreal amphotericin $\mathrm{B}(5 \mu \mathrm{g} / 0.1 \mathrm{~mL})$, known as the AmB-Vo regimen, until resolution of vitreous exudates. The decision to inject antifungals was based on detection of fungal hyphae on smears from corneal scrapings or clinical suspicion of fungal etiology. All post cataract surgery endophthalmitis cases underwent IOL explantation, seven eyes underwent repeat vitrectomy, and three eyes underwent penetrating keratoplasty. Systemic antifungal ketoconazole $200 \mathrm{mg}$ twice daily was administered in all cases with corneoscleral involvement. The mean duration of treatment with the AmB-Vo regimen was 21.5 (range 13-30) days. Patients with corneal or scleral involvement also received topical $5 \%$ natamycin and $1 \%$ voriconazole eye drops.

Globe salvage was achieved in all cases, with a final visual acuity of light perception or better. Visual acuity at last follow-up ranged from 20/60 to light perception, with 20/400 or better in seven of the 12 eyes (58.3\%) and 20/60 in 2/12 (16.6\%) eyes. All eyes had resolution of vitreous exudates as assessed by indirect ophthalmoscopy or by B-scan ultrasonography where the cornea was hazy. The mean presenting and final intraocular pressures were 9.5 (range 2-17) $\mathrm{mmHg}$ and 6.8 (range 3-10) $\mathrm{mmHg}$, respectively. Clinical pictures of the anterior segments from a few patients at presentation and the final visit are shown in Figures 1 and 2.

Culture-proven fungal endophthalmitis was seen in 11/12 eyes (91.6\%), while one post cataract surgery endophthalmitis case did not grow any organisms (Table 1, case 3). Fungal filaments were seen on direct examination of the vitreous sample (Calcofluor white and Gram stain) in 4/12 (33.3\%) cases and in corneal scrapings in 4/12 (33.3\%). Significant growth of filamentous fungi was seen in cultures for vitreous samples in 7/12 eyes (58.3\%) and explanted intraocular lens plated on chocolate agar in $7 / 12$ eyes $(58.3 \%$, Table 1$)$. Characteristic fungal colonies, either cinnamon brown, powdery, or yellowish green, granular were grown, which were suggestive of Aspergillus terreus and Aspergillus flavus, respectively. Lacto phenol cotton blue mount showed characteristic spores of both species. The vitreous from the single case of Fusarium endophthalmitis showed a creamy, fluffy pinkish colony and spores characteristic of Fusarium on microscopy.

\section{Discussion}

Fungal endophthalmitis is often refractory to antifungal therapy, with poor functional and anatomic outcomes. ${ }^{5,6}$ The principal objective of this study was to evaluate the role of combined intravitreal amphotericin B and voriconazole in the management of endophthalmitis caused by filamentous fungi.

In the current study, we treated filamentous fungal endophthalmitis with pars plana vitrectomy and a combination of intravitreal antifungal agents. This antifungal combination regimen was based on our clinical experience with case 1 (Table 1). This patient had undergone an open 


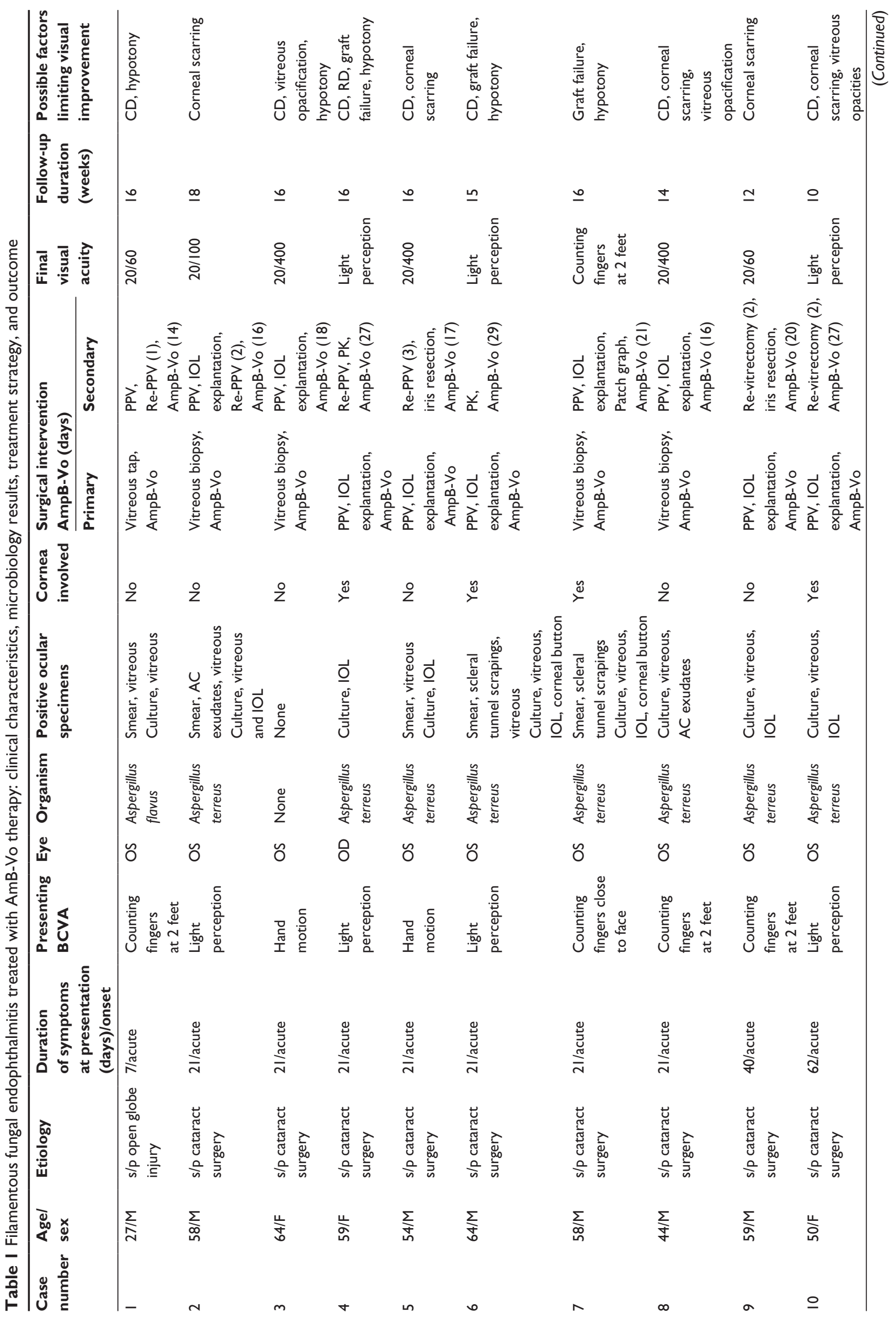




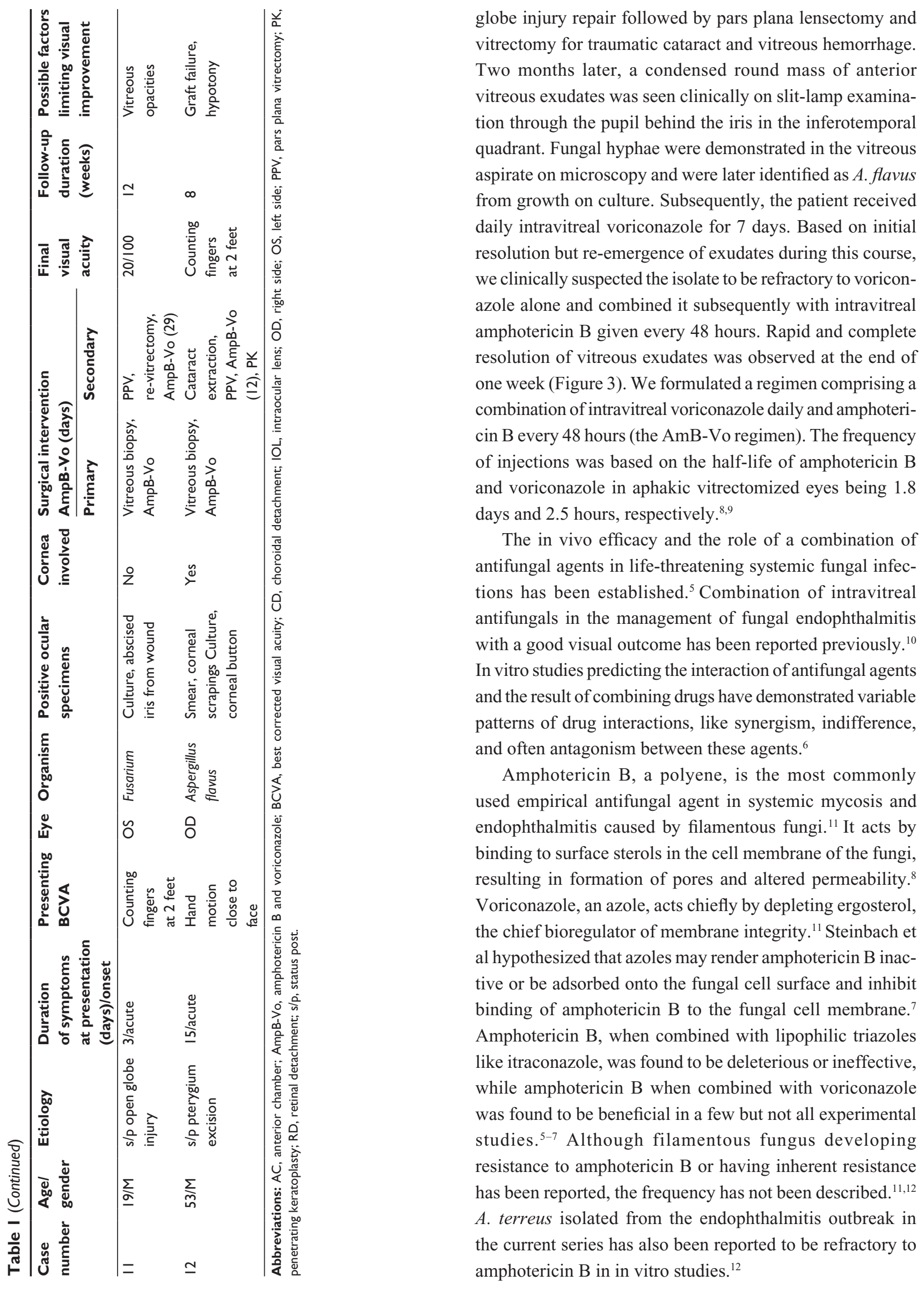

globe injury repair followed by pars plana lensectomy and morrhage. Two months later, a condensed round mass of anterior vitreous exudates was seen clinically on slit-lamp examinathe iris in the inferotemporal aspirate on microscopy and were later identified as A. flavus from growth on culture. Subsequently, the patient received daily intravitreal voriconazole for 7 days. Based on initial esolution but re-emergence of exudates during this course, we clinically suspected the isolate to be refractory to voriconazole alone and combined it subsequently with intravitreal amphotericin B given every 48 hours. Rapid and complete resolution of vitreous exudates was observed at the end of neek (Figure 3). We formulated a regimen comprising a combination of intravitreal voriconazole daily and amphotericin B every 48 hours (the AmB-Vo regimen). The frequency of injections was based on the half-life of amphotericin B and voriconazole in aphakic vitrectomized eyes being 1.8 ays and 2.5 hours, respectively. ${ }^{8,9}$

The in vivo efficacy and the role of a combination of intravitreal antifungals in the management of fungal endophthalmitis with a good visual outcome has been reported previously. ${ }^{10}$ variable patterns of drug interactions, like synergism, indifference, nd often antagonism between these agents. ${ }^{6}$

Amphotericin B, a polyene, is the most commonly mycosis and endophthalmitis caused by filamentous fungi. ${ }^{11}$ It acts by binding to surface sterols in the cell membrane of the fungi, resulting in formation of pores and altered permeability. ${ }^{8}$ perterol, he chief bioregulator of membrane integrity. ${ }^{11}$ Steinbach et al hypothesized that azoles may render amphotericin B inactive or be adsorbed onto the fungal cell surface and inhibit Amphotericin B, when combined with lipophilic triazoles like itraconazole, was found to be deleterious or ineffective, while amphotericin B when combined with voriconazole was found to be beneficial in a few but not all experimental studies. ${ }^{5-7}$ Although filamentous fungus developing resistance to amphotericin B or having inherent resistance has been reported, the frequency has not been described. ${ }^{11,12}$ outbreak in the current series has also been reported to be refractory to 

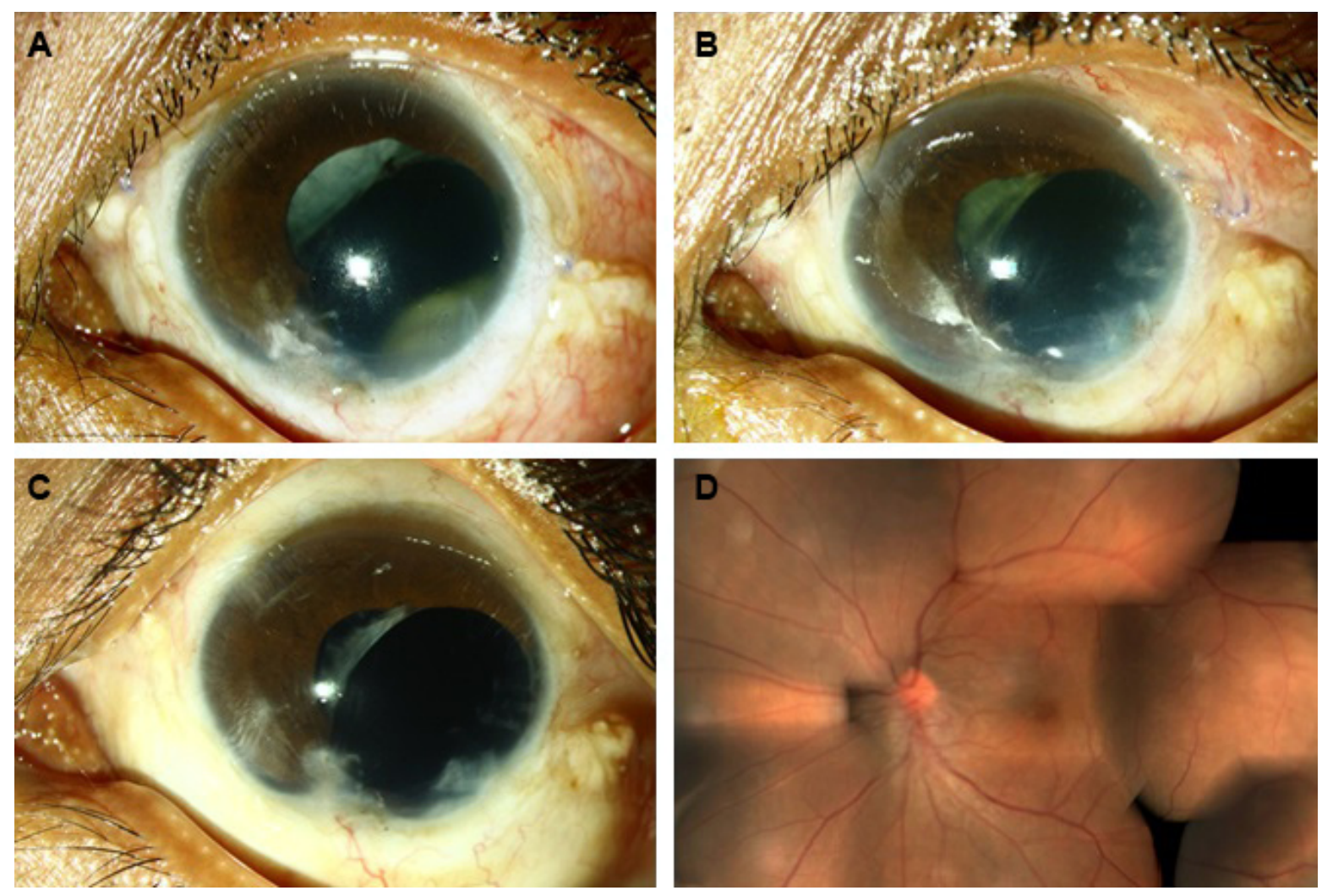

Figure I Clinical photographs of case I.

Notes: (A), ball of fungal exudates visible through pupil 2 months following open globe injury repair and vitrectomy. (B) partial response and reappearance of exudates following treatment with intravitreal voriconazole. (C), rapid and complete resolution of exudates with combined antifungal treatment observed at one week. (D), fundus photograph 3 months after resolution of endophthalmitis.
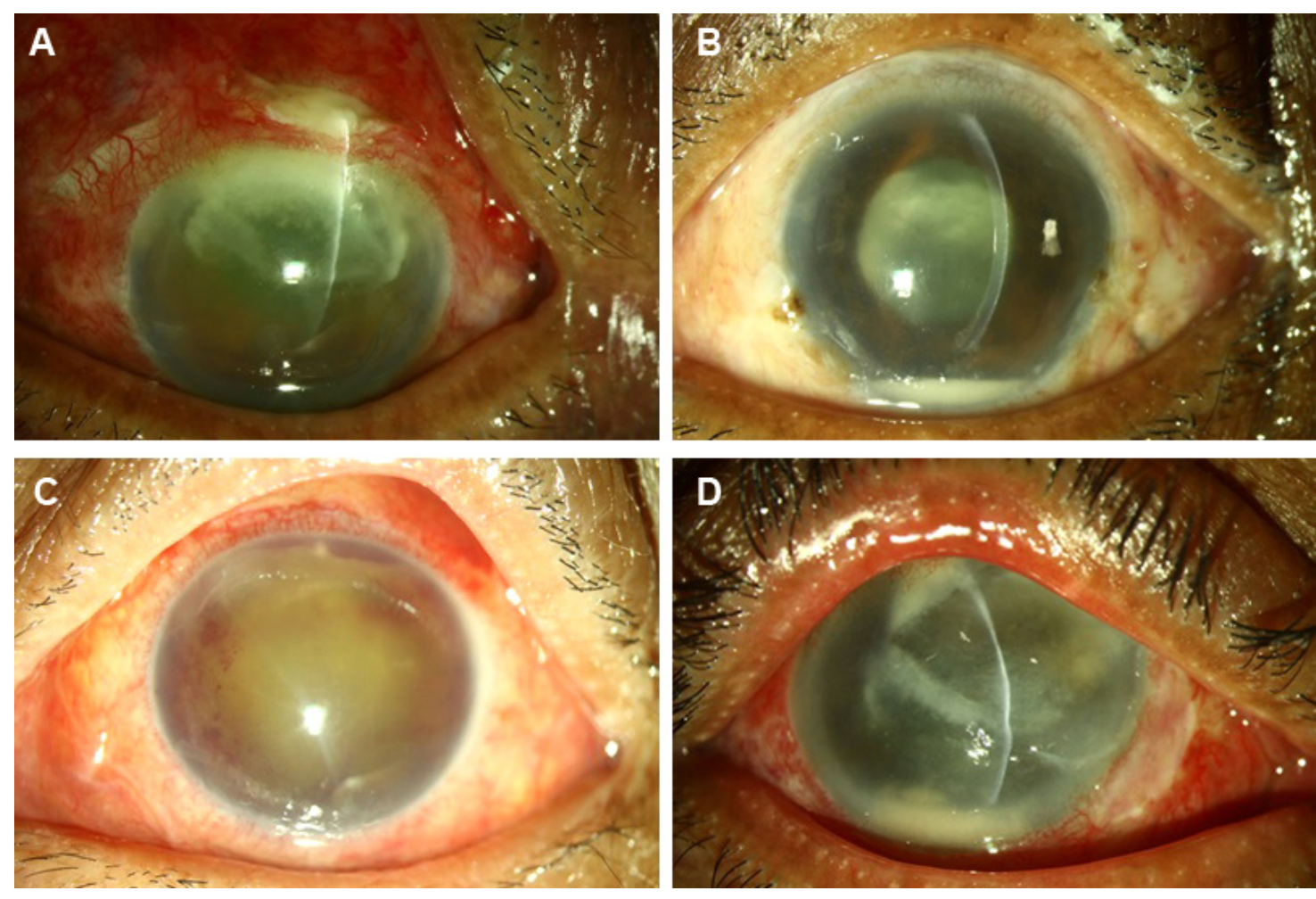

Figure 2 Preoperative clinical photographs of a few exogenous filamentous fungal endophthalmitis cases.

Notes: (A), post cataract surgery endophthalmitis with corneal and scleral tunnel fungal infiltrate. (B), cobweb-like exudates in pupillary area and on intraocular lens. (C), organized coagulum in the anterior chamber. (D), post-pterygium excision keratitis and endophthalmitis. 

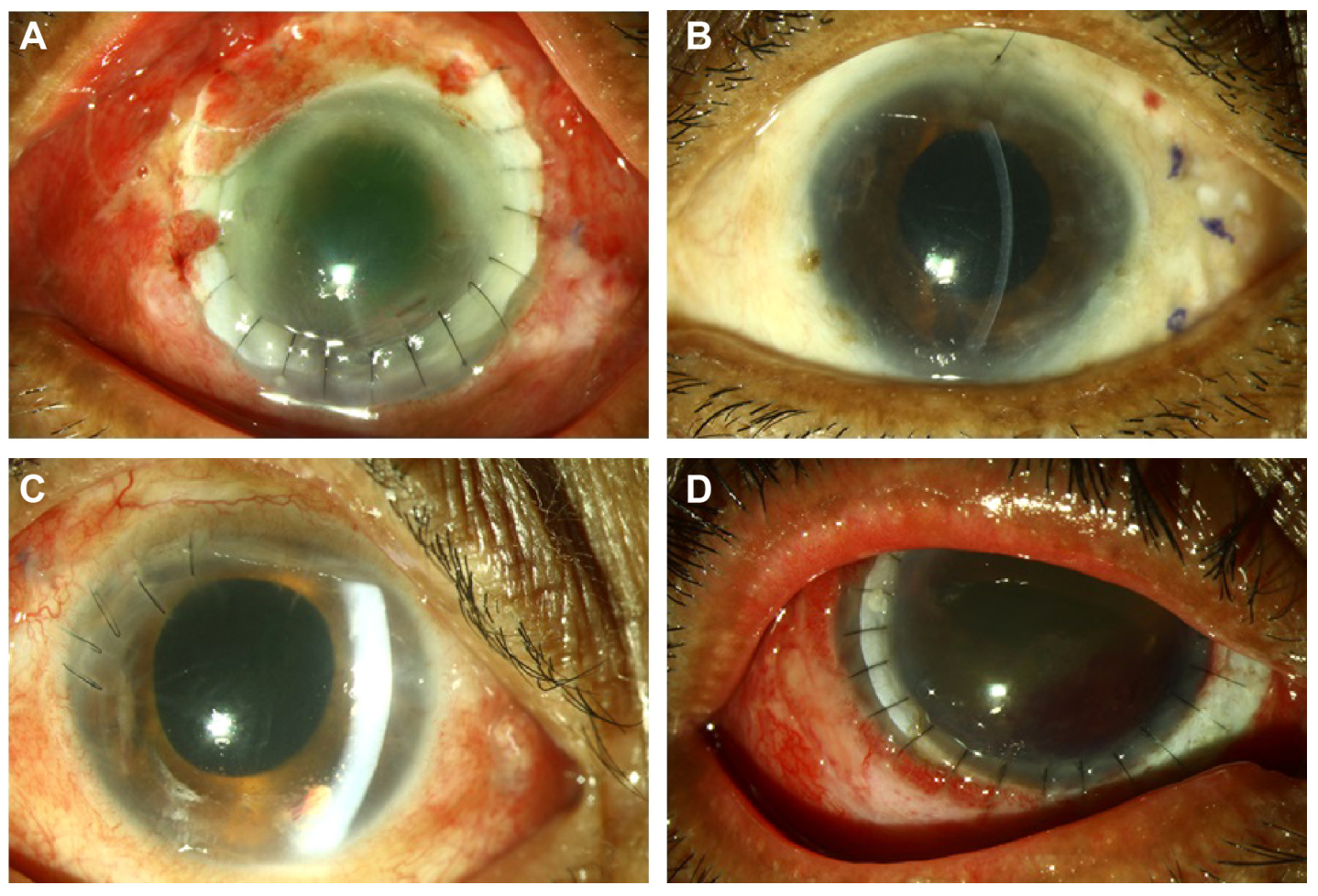

Figure 3 (A-D) Postoperative clinical photographs after resolution of endophthalmitis following vitrectomy, intravitreal antifungal combination therapy (amp-Vo regimen), and adjuvant procedures.

Wykoff et al have described fungal isolates, treatment strategies, and clinical outcomes in cases with exogenous fungal endophthalmitis. ${ }^{13}$ Systemic antifungals were required in $83 \%$ of patients, with additional intraocular antifungals needed in $95 \%$ of eyes. Fifty-nine percent of patients were treated with multiple antifungal agents and $20 \%$ received antifungal agents for 3-6 months. Fifty-four percent of patients achieved a final visual acuity of $20 / 400$ or better while $24 \%$ eyes lost perception of light including 20\% that underwent enucleation. Amongst the various categories, $54 \%$ of patients with postoperative fungal endophthalmitis achieved a visual acuity of 20/80 or better, while none of the cases with fungal endophthalmitis following open globe injury achieved vision better than $20 / 400$, and $70 \%$ of these underwent enucleation. Narang et al reported poor visual and anatomic outcomes in eyes with exogenous fungal endophthalmitis, especially when associated with corneal involvement. ${ }^{2}$

In the current study, at a mean final follow-up of 12.75 weeks, the best corrected visual acuity achieved was $20 / 400$ or better in $7 / 12$ eyes (58.33\%) and $20 / 60$ in $2 / 12$ eyes $(16.6 \%)$. None of the eyes lost light perception or were enucleated. All of the five eyes with corneal involvement developed hypotony and corneal haze or graft opacification with visual acuity of 20/400 or worse. Therapeutic penetrating keratoplasty was performed in three of these eyes. Five of six eyes with persistent hypotony had final visual acuity less than 20/400 at last follow-up, with only one such eye recovering a visual acuity of 20/60. Unlike previous reports of poor visual and anatomic outcomes in fungal endophthalmitis following open globe injury, ${ }^{13}$ patients in the current study recovered a visual acuity of $20 / 100$ or better at the last visit. Despite a delayed presentation (mean 27.6 days), $5 / 9(55.5 \%)$ patients who were part of a postoperative outbreak of endophthalmitis caused by $A$. terreus finally recovered visual acuity of 20/400 or better.

The main limitations of this study are its retrospective design and limited follow-up period. Further, all patients could not afford systemic antifungal drugs. Due to the paucity of such cases, a prospective randomized controlled trial would be difficult to perform, but a head-to-head comparison between voriconazole alone and a combination of voriconazole and amphotericin B would be useful for establishing which regimen is better. The majority (75\%) of isolates in the current series were A. terreus, which has previously been reported to be inherently resistant to amphotericin B. ${ }^{12}$ It would be useful to study the effect of combining amphotericin B and voriconazole in vitro with regard to possible synergism and correlate it with the in vivo response. 
In conclusion, combination intravitreal amphotericin B and voriconazole injections until complete resolution of vitreous exudates could be a promising modality of treatment in the management of exogenous filamentous fungus endophthalmitis.

\section{Disclosure}

The authors report no conflicts of interest in this work.

\section{References}

1. Callanan D, Scott IU, Murray TG, Oxford KW, Bowman CB, Flynn HW. Early onset endophthalmitis caused by Aspergillus species following cataract surgery. Am J Ophthalmol. 2006;142:509-511.

2. Narang S, Gupta A, Gupta V, et al. Fungal endophthalmitis following cataract surgery: clinical presentation, microbiological spectrum, and outcome. Am J Ophthalmol. 2001;132:609-617.

3. Walsh TJ, Peter J, McGough DA, Fothergill AW, Rinaldi MG, Pizzo PA. Activities of amphotericin B and antifungal azoles alone and in combination against Pseudallescheria boydii. Antimicrob Agents Chemother. 1995;39:1361-1364.

4. Walsh TJ, Petraitis V, Petraitiene R, et al. Experimental pulmonary aspergillosis due to Aspergillus terreus: pathogenesis and treatment of an emerging fungal pathogen resistant to amphotericin B. J Infect Dis. 2003;188:305-319.

5. Ostrosky-Zeichner L. Combination antifungal therapy: a critical review of the evidence. Clin Microbiol Infect. 2008;14 Suppl 4:65-70.
6. Clemons KV, Stevens DA. Animal models testing monotherapy versus combination antifungal therapy: lessons learned and future directions. Curr Opin Infect Dis. 2006;19:360-364.

7. Steinbach WJ, Stevens DA, Denning DW. Combination and sequential antifungal therapy for invasive aspergillosis: review of published in vitro and in vivo interactions and 6281 clinical cases from 1966 to 2001 Clin Infect Dis. 2003;37 Suppl 3:S188-S224.

8. Wingard LB Jr, Zuravleff JJ, Doft BH, Berk L, Rinkoff J. Intraocular distribution of intravitreally administered amphotericin B in normal and vitrectomized eyes. Invest Ophthalmol Vis Sci. 1989;30(10): 2184-2189.

9. Shen $\mathrm{Y}$, Wang M, Wang C, et al. Clearance of intravitreal voriconazole. Invest Ophthalmol Vis Sci. 2007;48:2238-2241.

10. Sheyman AT, Cohen BZ, Friedman AH, Ackert JM. An outbreak of fungal endophthalmitis after intravitreal injection of compounded combined bevacizumab and triamcinolone. JAMA Ophthalmol. 2013;131:864-869.

11. Ghannoum MA, Rice LB. Antifungal agents: mode of action, mechanism of resistance, and correlation of these mechanisms with bacterial resistance. Clin Microbiol Rev. 1999;12:501-517.

12. Sutton DA, Sanche SE, Revankar SG, Fothergill AW, Rinaldi MG. In vitro amphotericin B resistance in clinical isolates of Aspergillus terreus, with a head-to head comparison to voriconazole. J Clin Microbiol. 1999;37:2343-2345.

13. Wykoff CC, Flynn HW, Miller D, Scott IU, Alfonso EC. Exogenous fungal endophthalmitis: microbiology and clinical outcomes. Ophthalmology. 2008;115:1501-1507.
Clinical Ophthalmology

\section{Publish your work in this journal}

Clinical Ophthalmology is an international, peer-reviewed journa covering all subspecialties within ophthalmology. Key topics include: Optometry; Visual science; Pharmacology and drug therapy in eye diseases; Basic Sciences; Primary and Secondary eye care; Patient Safety and Quality of Care Improvements. This journal is indexed on

Submit your manuscript here: http://www.dovepress.com/clinical-ophthalmology-journal

\section{Dovepress}

PubMed Central and CAS, and is the official journal of The Society of Clinical Ophthalmology (SCO). The manuscript management system is completely online and includes a very quick and fair peer-review system, which is all easy to use. Visit http://www.dovepress.com/ testimonials.php to read real quotes from published authors. 